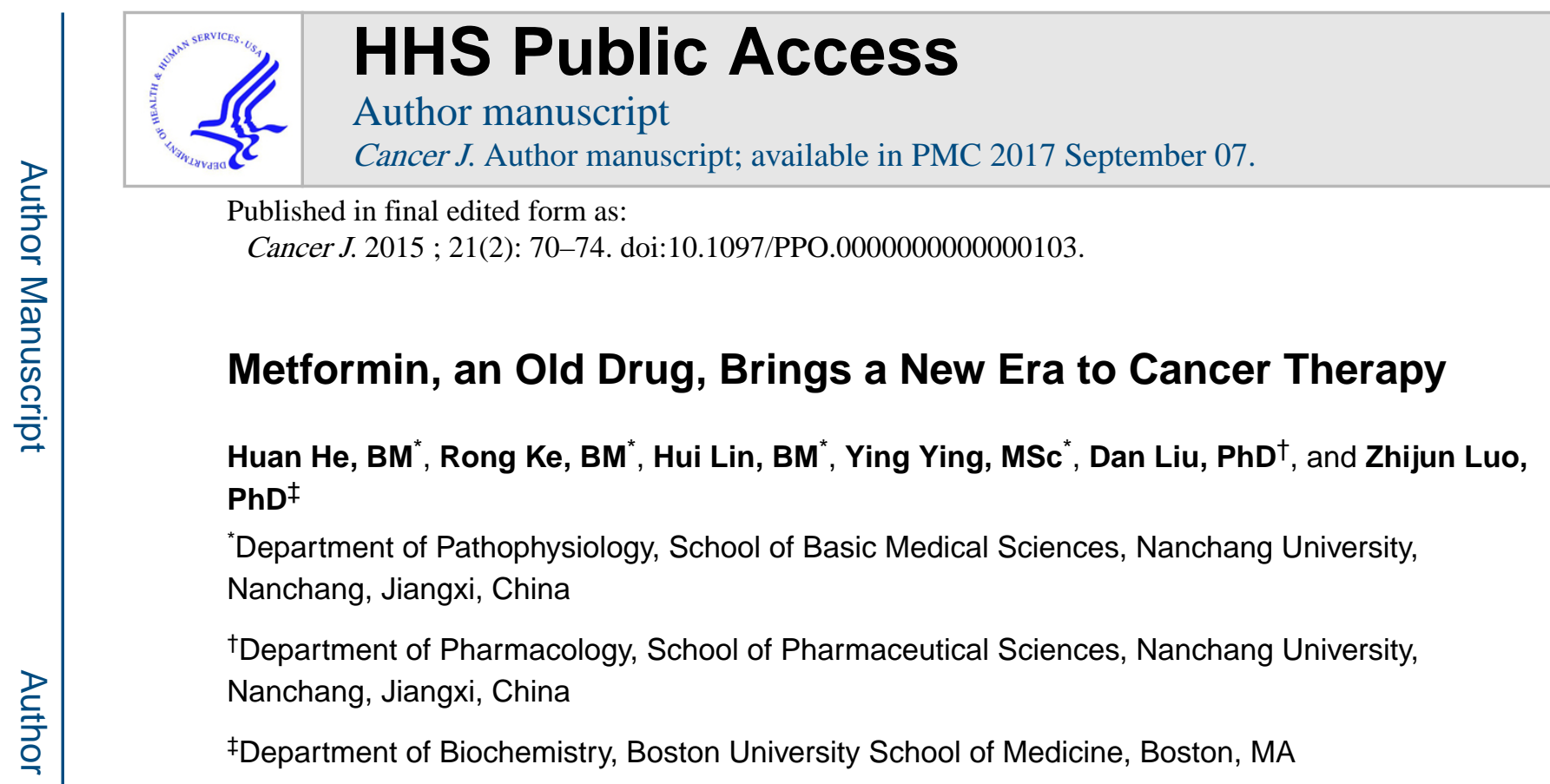

\begin{abstract}
Metformin is the most commonly prescribed drug for type 2 diabetes mellitus as it is inexpensive, safe, and efficient in ameliorating hyperglycemia and hyperinsulinemia. Epidemiological and clinical observation studies have shown that metformin use reduces risk of cancer in patients with type 2 diabetes mellitus and improves prognosis and survival rate of the cancer patients.

Furthermore, ongoing clinical trials of metformin in cancer therapy are extending to nondiabetic population. Thus, metformin is emerging as an attractive candidate for cancer prevention and treatment. This review summarizes recent progress in studies of metformin use in cancer and discusses the possibility to enhance its efficacy and to prevent cancer metastasis.
\end{abstract}

\title{
Keywords
}

Metformin; diabetes; cancer therapy

\begin{abstract}
Metformin is an antihyperglycemia drug and insulin sensitizer that improves insulin resistance and decreases fasting plasma insulin levels. ${ }^{1}$ Metformin also inhibits lipogenesis and mitigates hyperlipidemia, reduces cellular levels of reactive oxygen species, and downregulates proinflammatory cytokines. Dysregulation of these processes constitutes a risk factor of cancer. ${ }^{2}$ Thus, metformin is also a promising drug candidate for malignancy.

Cancer can be regarded as a metabolic disease. ${ }^{3}$ In 1920s, Warburg described that cancer cells utilize glycolysis as the major energy-producing mechanism for their survival even in the presence of oxygen, which is called aerobic glycolysis, also Warburg effect. ${ }^{4}$ This phenomenon of cancer cells is attributed to increased activity and expression of hypoxiainducing factor $1 a$, resulting in up-regulation of glucose transporter 1 and changes in enzymes for glycolysis and oxidative phoshorylation. ${ }^{5}$ In addition, other alterations in
\end{abstract}

Reprints: Zhijun Luo, PhD, Department of Biochemistry, Boston University, School of Medicine, 72 E. Concord Street, Boston, MA 02118. zluo@bu.edu.

The authors have disclosed that they have no significant relationships with, or financial interest in, any commercial companies pertaining to this article. 
metabolism are often associated with cancer; for instance, de novo synthesis of fatty acid is frequently found to be increased where enzymes such as fatty acid synthase (FASN) are upregulated, and protein synthesis is enhanced because of oncogenic activation of the mTOR pathway. ${ }^{2,6}$ Since a retrospective investigation in 2005 reporting that patients with type 2 diabetes mellitus (T2DM) receiving metformin displayed a significant decrease in cancer incidence than did those taking other glucose-lowering drugs, the number of epidemiological and experimental studies is rapidly increased on the effect of metformin as well as phenformin in prevention and treatment of primary and metastatic cancer. ${ }^{7}$ Therefore, in light of many excellent reviews on the topic of metformin and cancer, we wish to summarize recent progress in this area and focus on types of cancer and time window suitable for the use of metformin.

\section{Association Between Diabetes Mellitus and Cancer}

Diabetes can be complicated with cancer. The association between diabetes and cancer was first described 80 years ago by a Joslin clinician, who identified 256 cases of cancer in approximately 10,000 patients with diabetes. ${ }^{8}$ This was later confirmed by a large body of epidemiological studies, and now it is recognized that risk increases in diabetes, with the highest for liver, pancreas, and endometrial cancer ( 2 -fold) and the secondary for colorectal, breast2- and bladder cancer (1.2- to 1.5 -fold). ${ }^{8,9}$ No association between diabetes and lung cancer and an inverse association with prostate cancer were found. ${ }^{8,10}$

\section{Metformin Use and Cancer}

\section{Action of Metformin in Diabetes Mellitus}

Metformin, along with phenformin and buformin, belongs to biguanide family that is an active component in French lilac or Galega officinalis, oral hypoglycemic chemicals. ${ }^{11}$ These plants were first used for the relief of polyuria, a symptom of diabetes, in ancient Egypt and medieval Europe. The compounds were developed as therapeutic drugs for the management of diabetes in European countries in the 1950s. However, because of the toxicity of lactic acidosis caused by phenformin and buformin, only metformin was approved for the treatment of diabetes in Europe in 1958 and then in the United States in 1995.

Two mechanisms may account for the action of metformin. Firstly, metformin inhibits complex I of the mitochondrial respiratory chain, leading to blockade of oxidative phosphorylation and consequently an increase in adenosine monophosphate (AMP) or the ratio of AMP to ATP. ${ }^{12,13}$ Increased AMP acts as an allosteric regulator for several enzymes including fructose 1, 6-bisphoshatase, adenylate cyclase, and AMP-activated protein kinase (AMPK), funneling to inhibition of gluconeogenesis in the liver, which serves as the primary target organ. ${ }^{1}$ Secondly, Foretz et al ${ }^{14}$ have reported that metformin inhibits hepatic gluconeogenesis independent of AMPK. Likewise, recent studies by Madiraju et al ${ }^{15}$ have shown that metformin inhibits mitochondrial glycerophosphate dehydrogenase, resulting in alteration of cellular redox state, reduced conversion of lactate and glycerol to glucose, and thus decreased hepatic gluconeogenesis. The inhibition occurs at micromolar concentrations 
of metformin while the concentrations required for inhibition of complex I exceed $1 \mathrm{mM}$, which are supra-pharmacological levels.

\section{Prevention of Cancer by Metformin}

In early 1980s, Dilman et al ${ }^{16,17}$ gave phenformin to patients with breast and colon cancer to correct dysregulated metabolism, so as to achieve "metabolic rehabilitation." They found that both primary and metastatic tumors were suppressed. Likewise, in animal model, phenformin reduces DMBA-induced tumor by $80 \%$, and chronic treatment of female transgenic HER-2/neu mice significantly inhibits tumorigenesis of mammary gland and delays onset of the tumors. ${ }^{18,19}$

During the last decade, numerous epidemiological studies have been published with regard to the preventive and therapeutic effects of metformin on various cancers. The first was a case-control study that retrospectively reviewed the clinical records from 923 T2DM patients in the United Kingdom and found a $23 \%$ reduction in the risk of developing cancer. ${ }^{7}$ Since then, many studies further looked into the association between metformin use and the risk of site-specific cancers. ${ }^{8,20,21}$ For instance, a meta-analysis using 18 observational studies and 561,836 patients revealed that metformin use was associated with overall $27 \%$ reduction in the risk of developing all types of cancer. ${ }^{22}$ Strong associations between metformin use and reduced risk were found in cancers from liver, colorectal, pancreatic, stomach, and esophageal cancers, whereas no consistent results were found on cancers from breast, prostate, and lung. ${ }^{22,23}$

\section{Treatment of Cancer With Metformin}

The conclusions on the associations between metformin use and reduction of cancer risk are obtained mostly from T2DM patients. Furthermore, for cancer of which the risk is reduced, metformin also improves overall survival in patients, suggesting that metformin exerts a therapeutic effect. ${ }^{20,21}$ It was observed that the use of metformin as a neoadjuvant increased pathological complete response rates in breast cancer patients with diabetes, compared with either diabetic patients without metformin or nondiabetic patients ( $24 \%$ vs $8 \%$ vs $16 \%$ ), although no significant survival was improved. ${ }^{24} \mathrm{~A}$ smaller study on triple-negative breast cancer patients with diabetes exposed to metformin showed no significant improvement of overall survival, but a nonsignificant trend toward a lower risk of distant metastasis. ${ }^{25}$ Another study recruiting 1031 cases with T2DM and breast cancer reported that metformin treatment significantly improved 5-year survival rate. ${ }^{26}$ Diabetic patients with liver cancer receiving metformin displayed significantly improved prognosis and reduction in mortality. ${ }^{27,28}$ In addition, metformin treatment of T2DM has shown improvement of prognosis and overall survival or reduction in mortality of other cancers such as pancreatic cancer, colorectal cancer, ovarian cancer, prostate cancer, esophageal adenocarcinoma, and non-small cell lung cancer (NSCLC) ${ }^{20}$ To be noteworthy, controversial results were obtained with NSCLC. While longer progression-free survival was associated with metformin use in NSCLC patients with T2DM in some studies, poorer overall survival and metastatic cancer or no chemopreventive effect was observed in other studies. ${ }^{29-32}$ Reasons accounting for the discrepancy of these epidemiological studies are not clear but may reflect differences in methods used and multivariate settings. 
Because metformin is still a prescribed drug that is mostly limited on the market to the use of diabetes, insulin resistance, and obesity, its effect on cancer in nondiabetic population remains largely unknown. It was reported that metformin reduced fasting insulin, improved some metabolic parameters, decreased the number of Ki67-positive cells, and altered gene expression of molecules in mTOR and AMPK pathways in nondiabetic women with breast cancer. ${ }^{33,34}$ A randomized study showed that metformin in a small dose $(250 \mathrm{mg} / \mathrm{d})$ reduced colorectal aberrant crypt foci, a surrogate marker for colorectal cancer, by $40 \%$ in nondiabetic patients. ${ }^{35}$ A recent phase II clinical trial using metformin in combination with 5 -fluorouracil (5-FU) in the treatment of colorectal cancer showed a promising outcome in terms of disease stabilization. ${ }^{21}$ The definitive effect of metformin on nondiabetic cancer will await more clinical trial data.

\section{Limitations and Indications of Metformin Use in Cancer Dose}

For diabetes-associated cancer, metformin prosecutes anticancer function via a systemic but indirect effect, which is mediated by improving hyperglycemia and hyperinsulinemia (Fig. 1). Metformin also directly acts on cancer cells by inhibiting growth-promoting factors such as mTOR, SREBP-1, and FASN or activating some tumor suppressors. ${ }^{2}$ This direct effect occurs through both AMPK-dependent and independent mechanisms. ${ }^{1,36,37}$ Thus far, the majority of information on the direct effect of metformin is obtained from in vitro studies where the concentration usually falls within the range of 5 to $10 \mathrm{mM}$. This is much greater than the steady-state levels in plasma of patients with T2DM, which is around $10 \mu \mathrm{M}$ and can reach as high as $40 \mu \mathrm{M}$ when standard prescribed doses are given. ${ }^{12}$ In vitro studies have shown that metformin at such low concentrations is not sufficient to cause AMPK activation, although metabolic changes can be elicited. ${ }^{38}$ Then, it is a question as to whether such plasma concentrations can efficiently inhibit malignant growth of cancer cells in humans given the inhibitory effect of metformin is mediated by AMPK activation. In this scenario, addition of other activators with different mechanisms may circumvent the limitation of low doses; for example, salicylate binds to a site different from AMP and directly activates AMPK ${ }^{39-41}$ Combined use of metformin and salicylate may generate a synergistic effect. Other pharmacological agents that could be used together with metformin include resveratrol, berberine, statin, sorafinib, and so on. ${ }^{39,42,43}$

\section{Sites}

According to studies in mice, administration of metformin at $50 \mathrm{mg} / \mathrm{kg}$ per day results inmaximal concentration of 50 to $60 \mu \mathrm{M}$ in hepatic portal vein. ${ }^{44}$ The greatest accumulation occurs in the small intestine and secondly in the stomach, colon, kidney, and liver (>2-fold). Thus, it appears that metformin is accumulated in the digestive and secretion organs, suggesting tumor originated from these sites could be the first target for orally administered metformin. It will be of interest to determine differences in response of these and other relatively deep and distant sites to metformin. 


\section{Genetic Determinants for Metformin Sensitivity}

Previous studies have illustrated that cancer cells lacking functional LKB1 or p53 are more sensitive to metabolic catastrophe (cytotoxic effect) caused by glucose deprivation or phenformin/metformin. ${ }^{45,46}$ A recent study by Sabatini's laboratory has depicted that the sensitivity to glucose restriction or biguanides correlates to the capacity of oxidative phosphorylation. ${ }^{47}$ Thus, they constructed a short-hairpin RNA library to identify glucose utilization gene signature. Their results have revealed that genes conferring the sensitivity to low glucose or phenformin are those for oxidative phosphorylation, and mutations in complex I render cancer cells more sensitive to low glucose or phenformin. Hence, the glucose utilization gene signature and mutations in mtDNA-encoded complex I subunits may serve as biomarkers for identifying tumors that are particularly sensitive to biguanides. Such tumors are not negligible as the prevalence of truncating mutations in mtDNA-encoded oxidative phosphorylation components is reported to be as high as $16 \% .{ }^{48}$

\section{Targeting EMT/Cancer Stem Cells}

Cancer stem cells (CSCs) that are present in small number within the tumor mass confer chemotherapeutic resistance and are able to regenerate different types of cells, thereby causing relapse of the disease. A landmark publication by Hirsch et $\mathrm{al}^{49}$ has reported that CSCs are especially sensitive to metformin. In their hands, metformin at low doses (100-300 $\mu \mathrm{M}$ ) selectively killed CSCs, whereas these doses failed to affect nonstem cancer cells. Recent studies have shown that metformin/AMPK inhibits epithelial-to-mesenchymal transition (EMT) via multiple mechanisms including acting on Wnt/transforming growth factor $\beta /$ hedgehog/interleukin 6 signaling as well as miRNAs. ${ }^{50-53}$ As EMT plays a critical role in transition of CSCs and metastasis, these studies point that combination of metformin with conventional chemotherapeutic agents will enhance the efficacy of chemotherapy and prevent relapse and progression of cancer. This may be especially important under the circumstances where tumor is resected and no obvious metastatic indication is detected. In clinical practice, the chance of metastasis is not infrequent in the case that metastasis is not found in local lymph nodes, as tiny amount of circulating tumor cells with self-renewal properties may cause lethal metastatic disease later. At this situation, it is usually hard to judge if preventive chemotherapy is necessary. Hence, it would be wise to use no overt toxic metformin for the preventive treatment after surgical resection even in the absence of detectable metastasis.

\section{SUMMARY}

Metformin is the most prescribed, inexpensive, and safe anti-diabetic drug. It is now found to reduce the risk of many cancers in patients with T2DM and improve their prognosis and survival rate. The anticancer effect of metformin can be both systemic (i.e., in diabetesassociated cancer by improving hyperglycemia, hyperlipidemia, and hyperinsulinemia) and direct or local (e.g., cytostatic or cytotoxic) (Fig. 2). Combination of metformin with other nonprescribed drugs that can activate AMPK could be a safe and inexpensive approach to increase the efficiency. Furthermore, recent findings that metformin and AMPK suppress CSCs/EMT could ignite the use of metformin in prevention of metastasis prior to its indication. Currently, more than 190 clinical trials are ongoing in various cancers and even 
extending to nondiabetic cancers. Outcomes of these trials would hopefully bring a newera to cancer prevention and therapy. We are enthusiastically looking to the day to use metformin as a nonprescribed drug in cancer prevention.

\section{Acknowledgments}

This work was supported by the National Nature Science Foundation of China $(81171952,81272926)$ and in part by a National Institutes of Health grant (R21EY024388).

\section{References}

1. Pernicova I, Korbonits M. Metformin-mode of action and clinical implications for diabetes and cancer. Nat Rev Endocrinol. 2014; 10:143-156. [PubMed: 24393785]

2. Luo Z, Zang M, Guo W. AMPK as a metabolic tumor suppressor: control of metabolism and cell growth. Future Oncol. 1956; 6:457-470.

3. Hainaut P, Plymoth A. Cancer as a metabolic disease. Curr Opin Oncol. 2012; 24:56-57. [PubMed: 22143370]

4. Warburg O. On the origin of cancer cells. Science. 1956; 123:309-314. [PubMed: 13298683]

5. Jang M, Kim SS, Lee J. Cancer cell metabolism: implications for therapeutic targets. Exp Mol Med. 2013; 45:e45. [PubMed: 24091747]

6. Luo Z, Saha AK, Xiang X, et al. AMPK, the metabolic syndrome and cancer. Trends Pharmacol Sci. 2005; 26:69-76. [PubMed: 15681023]

7. Evans JM, Donnelly LA, Emslie-Smith AM, et al. Metformin and reduced risk of cancer in diabetic patients. BMJ. 2005; 330:1304-1305. [PubMed: 15849206]

8. Joost HG. Diabetes and cancer: epidemiology and potential mechanisms. Diab Vasc Dis Res. 2014; 11:390-394. [PubMed: 25268021]

9. Vigneri P, Frasca F, Sciacca L, et al. Diabetes and cancer. Endocr Relat Cancer. 2009; 16:11031123. [PubMed: 19620249]

10. Hall GC, Roberts CM, Boulis M, et al. Diabetes and the risk of lung cancer. Diabetes Care. 2005; 28:590-594. [PubMed: 15735193]

11. Bailey CJ, Day C. Avandamet: combined metformin-rosiglitazone treatment for insulin resistance in type 2 diabetes. Int J Clin Pract. 2004; 58:867-876. [PubMed: 15529521]

12. Owen MR, Doran E, Halestrap AP. Evidence that metformin exerts its anti-diabetic effects through inhibition of complex 1 of the mitochondrial respiratory chain. Biochem J. 2000; 348(pt 3):607614. [PubMed: 10839993]

13. Zhang L, He H, Balschi JA. Metformin and phenformin activate AMP-activated protein kinase in the heart by increasing cytosolic AMP concentration. Am J Physiol Heart Circ Physiol. 2007; 293:H457-H466. [PubMed: 17369473]

14. Foretz M, Hebrard S, Leclerc J, et al. Metformin inhibits hepatic gluconeogenesis in mice independently of the LKB1/AMPK pathway via a decrease in hepatic energy state. J Clin Invest. 2010; 120:2355-2369. [PubMed: 20577053]

15. Coughlan KA, Valentine RJ, Ruderman NB, et al. AMPK activation: a therapeutic target for type 2 diabetes? Diabetes Metab Syndr Obes. 2014; 7:241-253. [PubMed: 25018645]

16. Dilman VM, Berstein LM, Ostroumova MN, et al. Metabolic immunodepression and metabolic immunotherapy: an attempt of improvement in immunologic response in breast cancer patients by correction of metabolic disturbances. Oncology. 1982; 39:13-19. [PubMed: 7058042]

17. Dilman VM, Berstein LM, Yevtushenko TP, et al. Preliminary evidence on metabolic rehabilitation of cancer patients. Arch Geschwulstforsch. 1988; 58:175-183. [PubMed: 3415435]

18. Dilman VM, Berstein LM, Zabezhinski MA, et al. Inhibition of DMBA-induced carcinogenesis by phenformin in the mammary gland of rats. Arch Geschwulstforsch. 1978; 48:1-8. [PubMed: 418748] 
19. Anisimov VN, Berstein LM, Egormin PA, et al. Effect of metformin on life span and on the development of spontaneous mammary tumors in HER-2/neu transgenic mice. Exp Gerontol. 2005; 40:685-693. [PubMed: 16125352]

20. Morales DR, Morris AD. Metformin in cancer treatment and prevention. Annu Rev Med. 2015; 66:17-29. [PubMed: 25386929]

21. Miranda VC, Barroso-Sousa R, Glasberg J, et al. Exploring the role of metformin in anticancer treatments: a systematic review. Drugs Today (Barc). 2014; 50:623-640. [PubMed: 25313369]

22. Franciosi M, Lucisano G, Lapice E, et al. Metformin therapy and risk of cancer in patients with type 2 diabetes: systematic review. PLoS One. 2013; 8:e71583. [PubMed: 23936520]

23. Zhang P, Li H, Tan X, et al. Association of metformin use with cancer incidence and mortality: a meta-analysis. Cancer Epidemiol. 2013; 37:207-218. [PubMed: 23352629]

24. Jiralerspong S, Palla SL, Giordano SH, et al. Metformin and pathologic complete responses to neoadjuvant chemotherapy in diabetic patients with breast cancer. J Clin Oncol. 2009; 27:32973302. [PubMed: 19487376]

25. Bayraktar S, Hernadez-Aya LF, Lei X, et al. Effect of metformin on survival outcomes in diabetic patients with triple receptor-negative breast cancer. Cancer. 2012; 118:1202-1211. [PubMed: 21800293]

26. Hou G, Zhang S, Zhang X, et al. Clinical pathological characteristics and prognostic analysis of 1,013 breast cancer patients with diabetes. Breast Cancer Res Treat. 2013; 137:807-816. [PubMed: 23292119]

27. Currie CJ, Poole CD, Jenkins-Jones S, et al. Mortality after incident cancer in people with and without type 2 diabetes: impact of metformin on survival. Diabetes Care. 2012; 35:299-304. [PubMed: 22266734]

28. Chen TM, Lin CC, Huang PT, et al. Metformin associated with lower mortality in diabetic patients with early stage hepatocellular carcinoma after radiofrequency ablation. J Gastroenterol Hepatol. 2011; 26:858-865. [PubMed: 21251068]

29. Tan BX, Yao WX, Ge J, et al. Prognostic influence of metformin as first-line chemotherapy for advanced nonsmall cell lung cancer in patients with type 2 diabetes. Cancer. 2011; 117:51035111. [PubMed: 21523768]

30. Bodmer M, Becker C, Jick SS, et al. Metformin does not alter the risk of lung cancer: a casecontrol analysis. Lung Cancer. 2012; 78:133-137. [PubMed: 22959242]

31. Smiechowski BB, Azoulay L, Yin H, et al. The use of metformin and the incidence of lung cancer in patients with type 2 diabetes. Diabetes Care. 2013; 36:124-129. [PubMed: 22923670]

32. Mazzone PJ, Rai H, Beukemann M, et al. The effect of metformin and thiazolidinedione use on lung cancer in diabetics. BMC Cancer. 2012; 12:410. [PubMed: 22978440]

33. Goodwin PJ, Pritchard KI, Ennis M, et al. Insulin-lowering effects of metformin in women with early breast cancer. Clin Breast Cancer. 2008; 8:501-505. [PubMed: 19073504]

34. Hadad S, Iwamoto T, Jordan L, et al. Evidence for biological effects of metformin in operable breast cancer: a pre-operative, window-of-opportunity, randomized trial. Breast Cancer Res Treat. 2011; 128:783-794. [PubMed: 21655990]

35. Hosono K, Endo H, Takahashi H, et al. Metformin suppresses colorectal aberrant crypt foci in a short-term clinical trial. Cancer Prev Res (Phila). 2010; 3:1077-1083. [PubMed: 20810669]

36. Kalender A, Selvaraj A, Kim SY, et al. Metformin, independent of AMPK, inhibits mTORC1 in a rag GTPase-dependent manner. Cell Metab. 2010; 11:390-401. [PubMed: 20444419]

37. Li Y, Xu S, Mihaylova MM, et al. AMPK phosphorylates and inhibits SREBP activity to attenuate hepatic steatosis and atherosclerosis in diet-induced insulin-resistant mice. Cell Metab. 2011; 13:376-388. [PubMed: 21459323]

38. Zhou G, Myers R, Li Y, et al. Role of AMP-activated protein kinase in mechanism of metformin action. J Clin Invest. 2001; 108:1167-1174. [PubMed: 11602624]

39. Chen S, Zhu X, Lai X, et al. Combined cancer therapy with non-conventional drugs: all roads lead to AMPK. Mini Rev Med Chem. 2014; 14:642-654. [PubMed: 25138094]

40. Hawley SA, Fullerton MD, Ross FA, et al. The ancient drug salicylate directly activates AMPactivated protein kinase. Science. 2012; 336:918-922. [PubMed: 22517326] 
41. Din FV, Valanciute A, Houde VP, et al. Aspirin inhibits mTOR signaling, activates AMP-activated protein kinase, and induces autophagy in colorectal cancer cells. Gastroenterology. 2012; 142:1504.e3-1515.e3. [PubMed: 22406476]

42. Groenendijk FH, Mellema WW, van der Burg E, et al. Sorafenib synergizes with metformin in NSCLC through AMPK pathway activation. Int J Cancer. 2015; 136:1434-1444. [PubMed: 25080865]

43. Danzig MR, Kotamarti S, Ghandour RA, et al. Synergism between metformin and statins in modifying the risk of biochemical recurrence following radical prostatectomy in men with diabetes. Prostate Cancer Prostatic Dis. 2015; 18:63-68. [PubMed: 25403419]

44. Wilcock C, Bailey CJ. Accumulation of metformin by tissues of the normal and diabetic mouse. Xenobiotica. 1994; 24:49-57. [PubMed: 8165821]

45. Shackelford DB, Abt E, Gerken L, et al. LKB1 inactivation dictates therapeutic response of nonsmall cell lung cancer to the metabolism drug phenformin. Cancer Cell. 2013; 23:143-158. [PubMed: 23352126]

46. Buzzai M, Jones RG, Amaravadi RK, et al. Systemic treatment with the antidiabetic drug metformin selectively impairs p53-deficient tumor cell growth. Cancer Res. 2007; 67:6745-6752. [PubMed: 17638885]

47. Birsoy K, Possemato R, Lorbeer FK, et al. Metabolic determinants of cancer cell sensitivity to glucose limitation and biguanides. Nature. 2014; 508:108-112. [PubMed: 24670634]

48. Larman TC, DePalma SR, Hadjipanayis AG, et al. Spectrum of somatic mitochondrial mutations in five cancers. Proc Natl Acad Sci U S A. 2012; 109:14087-14091. [PubMed: 22891333]

49. Hirsch HA, Iliopoulos D, Tsichlis PN, et al. Metformin selectively targets cancer stem cells, and acts together with chemotherapy to block tumor growth and prolong remission. Cancer Res. 2009; 69:7507-7511. [PubMed: 19752085]

50. Zhao Z, Cheng X, Wang Y, et al. Metformin inhibits the IL-6-induced epithelial-mesenchymal transition and lung adenocarcinoma growth and metastasis. PLoS One. 2014; 9:e95884. [PubMed: 24789104]

51. Zhang J, Shen C, Wang L, et al. Metformin inhibits epithelial-mesenchymal transition in prostate cancer cells: involvement of the tumor suppressor miR30a and its target gene SOX4. Biochem Biophys Res Commun. 2014; 452:746-752. [PubMed: 25201727]

52. Chou CC, Lee KH, Lai IL, et al. AMPK reverses the mesenchymal phenotype of cancer cells by targeting the Akt-MDM2-Foxo3a signaling axis. Cancer Res. 2014; 74:4783-4795. [PubMed: 24994714]

53. Bao B, Azmi AS, Ali S, et al. Metformin may function as anti-cancer agent via targeting cancer stem cells: the potential biological significance of tumor-associated miRNAs in breast and pancreatic cancers. Ann Transl Med. 2014; 2:59. [PubMed: 25333034] 


\section{Systemic effects in DM}

\section{Cellular effects on cancer cells}

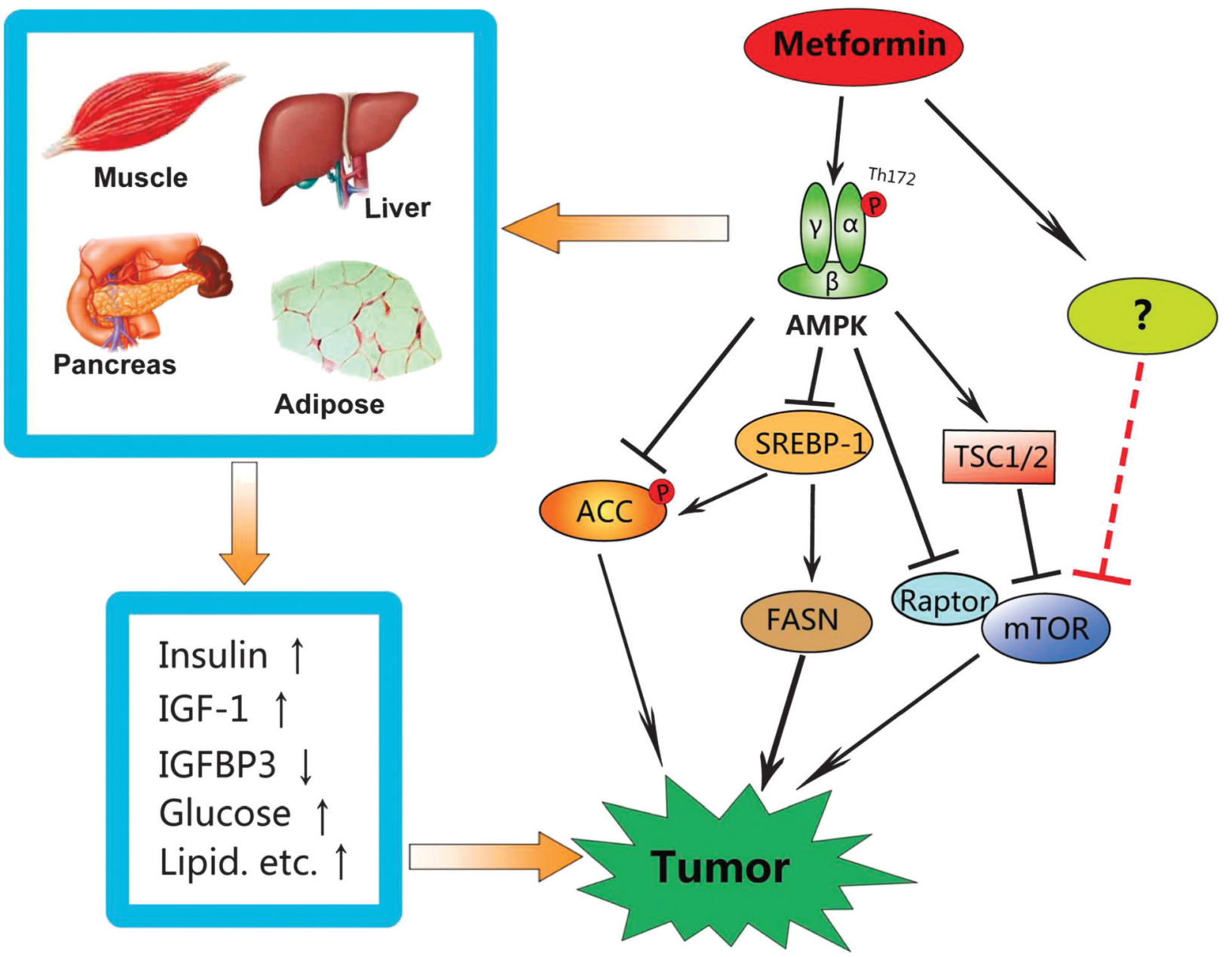

FIGURE 1.

Action of metformin on cancer. Two mechanisms are involved. First, in diabetes-associated cancer, metformin systemically ameliorates hyperinsulinemia, hypoglycemia, and hyperlipidemia, which are promoting factors not only for initiation of cancer, but also for progression of cancer. Second, at cellular levels, metformin activates AMPK, an immediate downstream effector of the tumor suppressor LKB1. Metformin inhibits the mTOR pathway through both AMPK-dependent and independent mechanisms. AMPK can also inhibit SREBP-1 by regulating its expression and phosphorylation, leading to down-regulation of FASN and acetyl-CoA carboxylase (ACC). In addition, AMPK directly phosphorylates and inhibits ACC. In summary, metformin suppresses de novo syntheses of fatty acids and protein synthesis in cancer cells. 


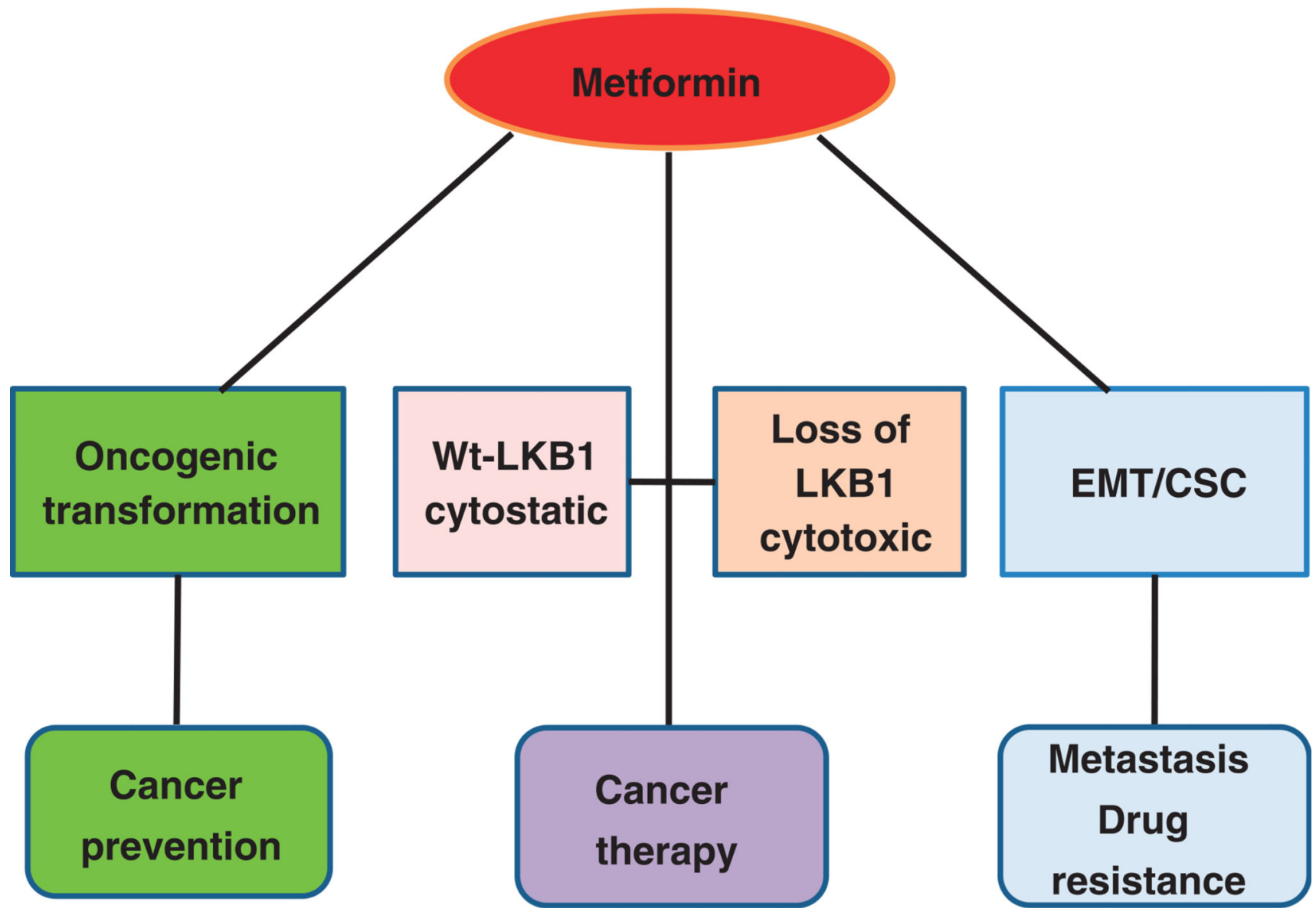

FIGURE 2.

Preventive and therapeutic role of metformin. (1) Metformin use can prevent malignant transformation and tumorigenesis via systemic and direct effect. (2) Metformin can act as an adjuvant or neoadjuvant agent. In cancer cells without functional LKB1 and inactivation of AMPK, metformin induces metabolic catastrophe and apoptosis (cytotoxic), while in the presence of LKB1, metformin activates AMPK, leading to inhibition of mTOR and cell cycle arrest (cytostatic). (3) Metformin suppresses EMT/CSCs, which can prevent metastasis and reverse drug resistance. 